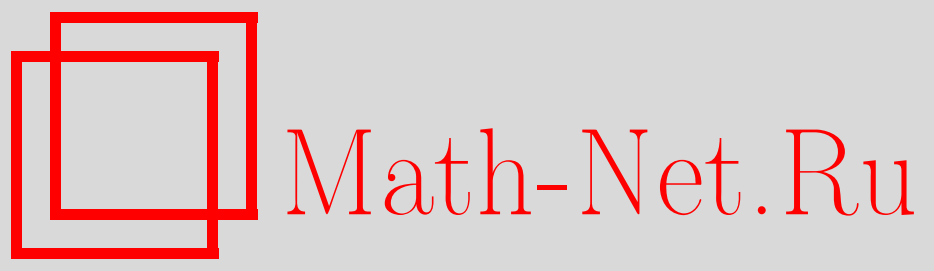

А. С. Либин, Крупномасштабные структуры как линии градиента: случай течения Тркаля, ТМФ, 2010, том 165, номер 2, 350-369

DOI: https://doi.org/10.4213/tmf6582

Использование Общероссийского математического портала Math-Net.Ru подразумевает, что вы прочитали и согласны с пользовательским соглашением http://www.mathnet.ru/rus/agreement

Параметры загрузки:

IP : 54.162 .85 .209

26 апреля 2023 г., 14:45:32

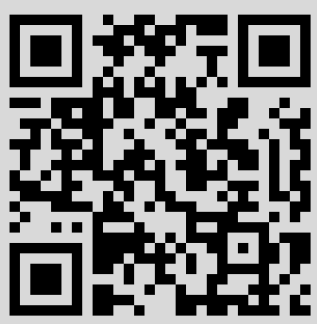




\title{
КРУПНОМАСШТАБНЫЕ СТРУКТУРЫ КАК ЛИНИИ ГРАДИЕНТА: СЛУЧАЙ ТЕЧЕНИЯ ТРКАЛЯ
}

\begin{abstract}
Методами мультискейлинга на базе членов разложения типа течения Бельтрами эффективно построено асимптотическое разложение при больших числах Рейнольдса для длинноволнового возмущения нестационарного анизотропного геликального решения нефорсированного уравнения Навье-Стокса (решения Тркаля). Доказано, что систематическая асимптотическая процедура может быть проведена лишь в случае, когда параметр скейлинга равен $R^{1 / 2}$. Проекции квазистационарных крупномасштабных линий тока на плоскость, ортогональную направлению анизотропии, оказываются линиями градиента функции, определяемой начальными условиями для двух модулированных (в результате скейлинга) анизотропных течений Бельтрами с одинаковыми собственными значениями оператора ротора, а трехмерные линии тока и линии ротора, не совпадая, заполняют инвариантные роторно-скоростные трубки, внутри которых векторы скорости и ротора коллинеарны с точностью до членов порядка $1 / R$.
\end{abstract}

Ключевые слова: крупномасштабные структуры, уравнения Навье-Стокса, течения Бельтрами, решения Тркаля, скоростная трубка, трубка ротора, линии градиента.

\section{1. ВВЕДЕНИЕ}

Для экспериментаторов когерентные структуры (KC) в турбулентном потоке проявляются как макроскопические подобласти когерентно движущихся объемов среди кажущегося хаоса. Несмотря на несомненную важность когерентных структур, процесс их возникновения остается неясным. На самом деле этот вопрос связан с более общим вопросом возникновения порядка в хаосе и из хаоса в ряде явлений природы.

Многие годы теория турбулентности шла в противоположном направлении. Со времени появления основопологающей работы Колмогорова, предложившего концепцию "прямого каскада" перекачки энергии на все уменьшающиеся характерные размеры вихрей, основное внимание уделялось статистическим концепциям "хаотизации" турбулентных потоков. Сами исходные уравнения Навье-Стокса фактически оставалась в тени.

Впервые идея о том, что когерентные структуры в развитом турбулентном потоке на деле являются областями, заполненными течениями типа Бельтрами, была

${ }^{*}$ E-mail: alexander.libin@gmail.com 
выдвинута в работах Левича с соавт. [1]-[4] и Моффата [5]-[7]. Отметим, что течения Бельтрами - анизотропные собственные вектор-функции оператора ротора являются стационарными решениями уравнений Эйлера, не содержащими вязких членов. Однако модифицированные течения Бельтрами, учитывающие их затухание в вязкой жидкости при отсутствии внешних сил ("нефорсированные уравнения Навье-Стокса") были найдены еще в 1918 г. Тркалем [8]. На сегодняшний день факт возникновения в развитой турбулентности областей геликальных течений типа Бельтрами, характеризуемых коллинеарностью векторов скорости и ротора скорости, твердо установлен в как геофизических наблюдениях, так и в численных симуляциях уравнений Навье-Стокса [9]-[18].

В свете этого в настоящей статье предполагается наличие в турбулентном потоке областей с течением типа Бельтрами. В целостной концепции, выдвинутой Левичем [3], идея подобластей с течениями типа Бельтрами количественно обобщается на основе экспериментальных данных геофизических наблюдений и результатов численных симуляций за последние 25 лет. Согласно этой концепции колмогоровский спектр K41 скорее всего порождается одним специальным классом таких подобластей с течением типа Бельтрами в них, встроенным в турбулентный поток. В основе этой концепции лежит идея ансамбля рождающихся и затухающих геликальных флюктуаций. Эти течения обладают большой геликальностью обоих знаков, и их можно рассматривать практически как течения Бельтрами. Замечательным свойством геликальных флюктуаций согласно Левичу оказывается малость четырехмерного фазового объема в пространстве-времени, связанная либо с малостью фрактального объема занимаемых ими подобластей, не превыщающего 2.5, либо с краткосрочностью их жизни. На протяжении времени жизни бельтрамиевских флюктуаций нелинейный член уравнений Навье-Стокса обращается в нуль, что приводит к общему уменьшению нелинейных взаимодействий во всем жидком объеме.

Однако возникающие и затухающие геликальные флюктуации никак не подходят на роль экспериментально наблюдаемых и практически неограниченно долго живущих КС.

Для выяснения математической природы КС мы используем идею и технику, предложенную Сивашинским [19], который рассматривал крупномасштабные структуры как проявление длинноволновой неустойчивости пространственно-периодических решений уравнений Навье-Стокса. Этот подход был применен в работах [20]-[23] для исследования нестабильности лннеаризованных уравнений Навье-Стокса, иногда с использованием приближений Галёркина, что потенциально могло снизить ценность полученного аналитического результата. В работе [24] исследовалась линейная устойчивость нестационарного решения Тркаля [8] нефорсированных уравнений Навье-Стокса и стационарного решения Бельтрами форсированных уравнений при больших значениях числа Рейнольдса $R$. Было установлено, что хотя линеаризованные форсирорванные уравнения Навье-Стокса неустойчивы к возмущениям с длиной волны $L$, пропорциональной $R$, нестационарные решения линеаризованных нефорсированных уравнений Навье-Стокса могут быть неустойчивы к возмущени- 
ям с некоторой промежуточной длиной волны $L$, меньшей $R$, причем порядок величины $L$ остается невыясненным.

В настоящей работе проведен нелинейный асимптотический анализ длинноволновых возмущений решения Тркаля для нефорсированного уравнения Навье-Стокса и эффективно построено асиптотическое решение, состоящее из течений типа Бельтрами и ассоциированных с ними членов, причем асимптотическая процедура оказывается осуществимой при единственном значении скейлингового параметра, равного $R^{1 / 2}$. Это позволяет выписать уравнения для линий тока, которые в квазистационарном случае оказываются градиентными линиями функции двух переменных, определяемой начальными условиями, и могут рассматриваться как крупномасштабные структуры. Критическим моментом всего анализа является спаривание двух крупномасштабно-модулированных анизотропных течений Бельтрами с одинаковым собственным значением оператора ротора.

Поразительным фактом является появление при анализе устойчивости скейлингового параметра, равного $R^{1 / 2}$ (связанного со специфическим выбором членов асимптотического разложения из течений Бельтрами), и сокращение на $R^{1 / 2}$ связывающей константы, стоящей перед нелинейным членом в ренормализованных разложениях в ряды теории возмущений, полученное в предположении доминирования флюктуаций Бельтрами в развитом турбулентном потоке [3].

\section{2. ВЫВОД АСИМПТОТИЧЕСКОГО РАЗЛОЖЕНИЯ}

Рассмотрим безразмерные нефорсированные уравнения Навье-Стокса для вязкой несжимаемой жидкости в ротационной форме:

$$
\begin{gathered}
\frac{\partial}{\partial t}(\operatorname{rot} \vec{u})+\operatorname{rot}[\operatorname{rot} \vec{u} \times \vec{u}]=\frac{1}{R} \Delta(\operatorname{rot} \vec{u}), \\
\operatorname{div} \vec{u}=0 .
\end{gathered}
$$

У этих уравнений есть хорошо известные решения Тркаля [8]

$$
\vec{u}_{0}(\vec{r}, t)=A e^{-\lambda^{2} t / R} \vec{h}(\vec{r}),
$$

где $\vec{h}(r)$ обозначает геликальное течение:

$$
\operatorname{rot} \vec{h}(\vec{r})=\lambda \vec{h}(r)
$$

Рассмотрим специфическое анизотропное геликальное течение с $\lambda=1$ (течение Бельтрами):

$$
\vec{h}_{0}(z)=\left(\begin{array}{c}
\sin z \\
\cos z \\
0
\end{array}\right), \quad \vec{u}_{0}(z, t)=A e^{-t / R} \vec{h}_{0}(z)
$$

Очевидно, что

$$
\operatorname{rot} \vec{h}_{0}(z)=\vec{h}_{0}(z) .
$$

Задача состоит в исследовании длинноволновой неустойчивости этого решения уравнения (1) при больших значениях числа Рейнольдса $R \gg 1$. 
Пусть $\vec{u}=\vec{V}+\vec{u}_{0}$. Тогда система уравнений для $V$ выглядит следующим образом:

$$
\frac{\partial}{\partial t}(\operatorname{rot} \vec{V})+A e^{-t / R} \operatorname{rot}\left[(\operatorname{rot} \vec{V}-\vec{V}) \times \vec{h}_{0}(z)\right]+\operatorname{rot}[\operatorname{rot} \vec{V} \times \vec{V}]=\frac{1}{R} \Delta \operatorname{rot} \vec{V},
$$

$$
\operatorname{div} \vec{V}=0, \quad R \gg 1
$$

Мы будем искать медленно эволюционирующие крупномасштабные модуляции для $\vec{V}$. Для этого, следуя работе [19], проделаем формальные скейлинговые процедуры:

$$
\begin{aligned}
\vec{V}(x, y, z, t) & \rightarrow \vec{V}(x, y, z, \xi, \eta, \theta, \tau), & & \\
\frac{\partial}{\partial x} & \rightarrow \frac{\partial}{\partial x}+\varepsilon \frac{\partial}{\partial \xi}, & & \xi \sim \varepsilon x, \\
\frac{\partial}{\partial y} & \rightarrow \frac{\partial}{\partial y}+\varepsilon \frac{\partial}{\partial \eta}, & & \eta \varepsilon y, \\
\frac{\partial}{\partial t} & \rightarrow \varepsilon \frac{\partial}{\partial \tau}, & & \tau \sim \varepsilon t,
\end{aligned}
$$

где $\varepsilon-$ малый параметр. Отсюда

$$
\begin{aligned}
& \operatorname{rot}_{x y z} \rightarrow \operatorname{rot}_{x y z}+\varepsilon \operatorname{rot}_{\xi \eta \theta}, \\
& \operatorname{div}_{x y z} \rightarrow \operatorname{div}_{x y z}+\varepsilon \operatorname{div}_{\xi \eta \theta} .
\end{aligned}
$$

Мы будем искать решения скейлинговых уравнений, не зависящие от $x, y$. Тогда система (2) принимает вид

$$
\begin{aligned}
& A e^{-\varepsilon \tau} \operatorname{rot}_{z}\left[\left(\operatorname{rot}_{z} \vec{V}-\vec{V}\right) \times \vec{h}_{0}\right]+\operatorname{rot}_{z}\left[\operatorname{rot}_{z} \vec{V} \times \vec{V}\right]+ \\
& \quad+\varepsilon\left\{A e^{-\varepsilon \tau}\left\{\operatorname{rot}_{z}\left[\operatorname{rot}_{\xi \eta} \vec{V} \times \vec{h}_{0}\right]+\operatorname{rot}_{\xi \eta}\left[\left(\operatorname{rot}_{z} \vec{V}-\vec{V}\right) \times \vec{h}_{0}\right]\right\}+\right. \\
& \left.+\operatorname{rot}_{\xi \eta}\left[\operatorname{rot}_{z} \vec{V} \times \vec{V}\right]+\operatorname{rot}_{z}\left[\operatorname{rot}_{\xi \eta} \vec{V} \times \vec{V}\right]+\frac{\partial}{\partial \tau}\left(\operatorname{rot}_{z} \vec{V}\right)-\frac{1}{R} \operatorname{rot}_{\xi \eta} \Delta_{z} \vec{V}\right\}+ \\
& +\varepsilon^{2}\left\{\operatorname{rot}_{\xi \eta}\left[\operatorname{rot}_{\xi \eta} \vec{V} \times \vec{V}\right]+A e^{-\varepsilon \tau} \operatorname{rot}_{\xi \eta}\left[\operatorname{rot}_{\xi \eta} \vec{V} \times \vec{h}_{0}\right]+\frac{\partial}{\partial \tau}\left(\operatorname{rot}_{\xi \eta} \vec{V}\right)+\right. \\
& \left.+\frac{1}{R} \operatorname{rot}_{z} \Delta_{\xi \eta} \vec{V}\right\}-\varepsilon^{3} \frac{1}{R} \Delta_{\xi \eta} \operatorname{rot}_{\xi \eta} \vec{V}-\frac{1}{R} \Delta_{z} \operatorname{rot}_{z} \vec{V}=0, \\
& \quad \operatorname{div}_{z} \vec{V}+\varepsilon \operatorname{div}_{\xi \eta} \vec{V}=0 .
\end{aligned}
$$

Поскольку $(1 / R) \Delta_{z} \operatorname{rot}_{z} V$ должен быть одним из членов первого из уравнений в (4), то для некоторого целого $k$ должно выполняться $\varepsilon^{k}=R^{-1}$ или $\varepsilon=R^{-1 / k}$. Мы будем искать решения системы (4) в виде асимптотического разложения по степеням $\varepsilon$ :

$$
\vec{V} \sim \vec{V}_{0}+\varepsilon \vec{V}_{1}+\varepsilon^{2} \vec{V}_{2}+\cdots
$$

Пусть

$$
\vec{h}_{1}=\left(\begin{array}{c}
\cos z \\
-\sin z \\
0
\end{array}\right) \text {. }
$$

6 Теоретическая и математическая физика, т. 165, № 2, 2010 г. 
Очевидно, что $\operatorname{rot} \vec{h}_{1}=\vec{h}_{1}$. Не существует никаких других линейно независимых $2 \pi$-периодических по $z$ течений, являющихся собственными векторами оператора ротора с собственным значением, равным 1 , кроме $\vec{h}_{0}$ и $\vec{h}_{1}$. Мы будем искать члены асимптотического разложения (5) в виде

$$
\vec{V}_{n}=\gamma_{0}^{(n)}(\xi, \eta, \tau) \vec{h}_{0}(z)+\gamma_{1}^{(n)}(\xi, \eta, \tau) \vec{h}_{1}(z)+\vec{\vartheta}_{n}(\xi, \eta, z, \tau)+\vec{\delta}_{n}(\xi, \eta, \tau)=\vec{w}_{n}+\vec{\vartheta}_{n}+\vec{\delta}_{n},
$$

где

$$
\vec{\vartheta}_{n}=\left(\begin{array}{c}
0 \\
0 \\
B_{n} \cos z+C_{n} \sin z
\end{array}\right), \quad \vec{\delta}=\left(\begin{array}{c}
0 \\
0 \\
\delta_{n}(\xi, \eta, \tau)
\end{array}\right)
$$

Очевидно, что

$$
\begin{gathered}
\operatorname{rot}_{z} \vec{\vartheta}_{n}=0, \\
\operatorname{rot}_{z} V_{n}=\operatorname{rot}_{z}\left[\gamma_{0}^{(n)} \vec{h}_{0}(z)+\gamma_{1}^{(n)} \vec{h}_{1}(z)\right]=\operatorname{rot}_{z} \vec{w}_{n}=\vec{w}_{n} .
\end{gathered}
$$

Разложение (5) в виде (6) будет эффективно построено ниже.

Поскольку мы ищем $2 \pi$-периодические по $z$ решения системы (4), можно проинтегрировать ее по периоду:

$$
\begin{aligned}
\operatorname{rot}_{\xi \eta} \int_{0}^{2 \pi} & \left\{A e^{-\varepsilon \tau}\left[\left(\operatorname{rot}_{z} \vec{V}-\vec{V}\right) \times \vec{h}_{0}\right]+\left[\operatorname{rot}_{z} \vec{V} \times \vec{V}\right]\right\} d z+ \\
& +\varepsilon \operatorname{rot}_{\xi \eta} \int_{0}^{2 \pi}\left\{\left[\operatorname{rot}_{\xi \eta} \vec{V} \times\left(\vec{V}+A e^{-\varepsilon \tau} \vec{h}_{0}\right)\right]+\frac{\partial \vec{V}}{\partial \tau}\right\} d z- \\
& -\varepsilon^{4} \Delta_{\xi \eta} \operatorname{rot}_{\xi \eta} \int_{0}^{2 \pi} \vec{V} d z=0 .
\end{aligned}
$$

На характерных временах $\tau \sim 1, t \sim \sqrt{R}$ мы "заморозим":

$$
e^{-\varepsilon \tau}=b_{0} \simeq 1 \text {. }
$$

Будем искать $\vec{V}_{0}$ и $\vec{V}_{1}$ в виде (6). Для $\varepsilon^{0}$ из (4) получаем

$$
\begin{gathered}
\operatorname{rot}_{z}\left[\operatorname{rot}_{z} \vec{V}_{0} \times \vec{V}_{0}\right]+A b_{0} \operatorname{rot}_{z}\left[\left(\operatorname{rot}_{z} \vec{V}_{0}-\vec{V}_{0}\right) \times \vec{h}_{0}\right]=0, \\
\operatorname{div}_{z} \vec{V}_{0}=0 .
\end{gathered}
$$

Легко видеть, что

$$
\vec{\vartheta}_{0}=\vec{\delta}_{0}=0
$$

поэтому

$$
\vec{V}_{0}(\xi, \eta, z, \tau)=\gamma_{0}^{(0)}(\xi, \eta, \tau) \vec{h}_{2}(z)+\gamma_{0}^{(1)}(\xi, \eta, \tau) \vec{h}_{1}(z) .
$$

Следовательно,

$$
\begin{gathered}
\operatorname{div}_{z} \vec{V}_{1}=-\operatorname{div}_{\xi \eta} \vec{V}_{0} \\
\operatorname{div}_{z} \vec{V}_{1}=\frac{d}{d z}\left(B_{1} \cos z+C_{1} \sin z\right)=C_{1} \cos z-B_{1} \sin z \\
\operatorname{div}_{\xi \eta} \vec{V}_{0}=\left(\frac{\partial \gamma_{0}^{(0)}}{\partial \xi}-\frac{\partial \gamma_{1}^{(0)}}{\partial \eta}\right) \sin z+\left(\frac{\partial \gamma_{0}^{(0)}}{\partial \eta}-\frac{\partial \gamma_{1}^{(0)}}{\partial \xi}\right) \cos z,
\end{gathered}
$$


T. e.

$$
B_{1}=\left(-\frac{\partial \gamma_{1}^{(0)}}{\partial \eta}+\frac{\partial \gamma_{0}^{(0)}}{\partial \xi}\right), \quad C_{1}=-\left(\frac{\partial \gamma_{0}^{(0)}}{\partial \eta}+\frac{\partial \gamma_{1}^{(0)}}{\partial \xi}\right)
$$

Итак,

$$
\vec{\vartheta}_{1}=\left(\begin{array}{c}
0 \\
0 \\
B_{1} \cos z+C_{1} \sin z
\end{array}\right)=\operatorname{rot}_{\xi \eta}\left(\gamma_{0}^{(0)} \vec{h}_{0}(z)+\gamma_{1}^{(0)} \vec{h}_{0}(z)\right) .
$$

Поскольку очевидно, что уравнение (8) выполняется для $\vartheta_{n+1}, \gamma_{0}^{(n)}, \gamma_{1}^{(n)}, n \geqslant 1$, можно построить асимптотическое разложение.

Пусть

$$
\begin{gathered}
\vec{V}_{1}=\vec{w}_{1}+\operatorname{rot}_{\xi \eta} \vec{V}_{0}+\vec{\delta}_{1}, \\
\operatorname{rot}_{z} \vec{V}_{1}=\operatorname{rot}_{z} \vec{w}_{1}=\vec{w}_{1}, \\
\vec{\delta}_{1}=\left(\begin{array}{c}
0 \\
0 \\
\delta_{1}(\xi, \eta, \tau)
\end{array}\right) .
\end{gathered}
$$

Подстановка $\vec{V}_{0}$ и $\vec{V}_{1}$ в таком виде в коэффициенты членов разложения (4) при $\varepsilon^{1}$ и $\varepsilon^{2}$ дает

$$
\frac{\partial \vec{V}_{0}}{\partial \tau}=\delta_{1} \gamma_{1}^{(0)} \vec{h}_{0}-\delta_{1}\left(A b_{0}+\gamma_{1}^{(0)}\right) \vec{h}_{1}
$$

или

$$
\begin{gathered}
\frac{\partial \gamma_{0}^{(0)}}{\partial \tau}=\delta_{1} \gamma_{1}^{(0)} \\
\frac{\partial \gamma_{1}^{(0)}}{\partial \tau}=-\delta_{1}\left(A b_{0}+\gamma_{0}^{(0)}\right)
\end{gathered}
$$

Следовательно,

$$
\frac{1}{2} \frac{\partial}{\partial \tau}\left\{\left(\gamma_{0}^{(0)}+A b_{0}\right)^{2}+\left(\gamma_{1}^{(0)}\right)^{2}\right\}=0
$$

или

$$
\left(\gamma_{0}^{(0)}+A b_{0}\right)^{2}+\left(\gamma_{1}^{(0)}\right)^{2}=\mathrm{const}=C_{0}^{2}(\xi, \eta)
$$

Отсюда

$$
\begin{gathered}
\gamma_{0}^{(0)}+A b_{0}=C_{0}(\xi, \eta) \cos \varphi(\xi, \eta, \tau) \\
\gamma_{1}^{(0)}=C_{0}(\xi, \eta) \sin \varphi(\xi, \eta, \tau) \\
\delta_{1}=-\frac{\partial \varphi}{\partial \tau}
\end{gathered}
$$

Итак, согласно уравнению (12) должен возникнуть вертикальный поток, скорость которого определяется эволюцией $\varphi$. 
Для получения эволюционного уравнения для $\delta_{1}$ (т. е. и для $\varphi$ ) нужно подставить в уравнение (7) следующие выражения:

$$
\begin{aligned}
& \vec{V}_{2}=\vec{w}_{2}+\operatorname{rot}_{\xi \eta} \vec{w}_{1}+\vec{\delta}_{2}, \\
& \vec{V}_{1}=\vec{w}_{1}+\operatorname{rot}_{\xi \eta} \vec{V}_{0}+\vec{\delta}_{1}, \\
& \vec{V}_{0}=\gamma_{0}^{(0)} \vec{h}_{1}+\gamma_{1}^{(0)} \vec{h}_{2} .
\end{aligned}
$$

Получаем

$$
\frac{\partial \vec{\delta}_{1}}{\partial \tau}=-\frac{1}{2}\left[\operatorname{rot}_{\xi \eta} \operatorname{rot}_{\xi \eta} \vec{V}_{0} \times\left(\vec{V}_{0}+A b_{0} \vec{h}_{0}\right)\right]
$$

однако

$$
\begin{gathered}
\operatorname{rot}_{\xi \eta} \operatorname{rot}_{\xi \eta} \vec{V}_{0}=-\left(\Delta_{\xi \eta} \gamma_{0}^{(0)}\right) \vec{h}_{0}-\left(\Delta_{\xi \eta} \gamma_{1}^{(0)}\right) \vec{h}_{1} \\
{\left[\vec{h}_{0} \times \vec{h}_{1}\right]=\left(\begin{array}{c}
0 \\
0 \\
-1
\end{array}\right)}
\end{gathered}
$$

В результате приходим к уравнению

$$
\frac{\partial \delta_{1}}{\partial \tau}=\frac{1}{2}\left[\left(A b_{0}+\gamma_{0}^{(0)}\right) \Delta_{\xi \eta} \gamma_{1}^{(0)}\right]-\frac{1}{2}\left[\gamma_{1}^{(0)} \Delta_{\xi \eta} \gamma_{0}^{(0)}\right]
$$

Учитывая уравнения (11), получаем

$$
\begin{gathered}
\frac{\partial^{2} \varphi}{\partial \tau^{2}}=-\frac{C_{0}^{2}(\xi, \eta)}{2} \Delta_{\xi, \eta} \varphi+\left(\frac{\partial C_{0}}{\partial \xi} \frac{\partial \varphi}{\partial \xi}+\frac{\partial C_{0}}{\partial \eta} \frac{\partial \varphi}{\partial \eta}\right) \\
C_{0}^{2}(\xi, \eta)=\left(A b_{0}+\gamma_{0}^{(0)}(\xi, \eta, 0)\right)^{2}+\left(\gamma_{1}^{(0)}(\xi, \eta, 0)\right)^{2} \\
\varphi(\xi, \eta, 0)=\operatorname{arctg} \frac{\gamma_{1}^{(0)}(\xi, \eta, 0)}{A b_{0}+\gamma_{0}^{(0)}(\xi, \eta, 0)} \\
\left.\frac{\partial \varphi}{\partial \tau}\right|_{\tau=0}=\delta_{1}(\xi, \eta, 0) \equiv \delta_{0}(\xi, \eta)
\end{gathered}
$$

Мы получили задачу Коши (с периодическими граничными условиями) для эллиптического уравнения - классический пример некорректно поставленной задачи, что указывает также на неустойчивость во времени.

Поскольку члены при $\varepsilon^{0}$ и $\varepsilon^{1}$ в системе (4) порождены только членами из левых частей уравнений (1) или (2), речь идет о неустойчивости Эйлера.

Для нахождения $\vec{w}_{1}$ (первый член в $\vec{V}_{1}$ ) нужно подставить члены разложения (6) при $n=0,1,2$ в систему (4), однако предварительно необходимо выяснить, для какого целого $\kappa$ выполняется равенство $\varepsilon^{\kappa}=R^{-1}$.

Предположим сначала, что $\kappa>2$. Тогда для $\varepsilon^{2}$-приближения получим

$$
\frac{\partial \vec{w}_{1}}{\partial \tau}+\left[\vec{w}_{1} \times \vec{\delta}_{1}\right]+\left[\left(\vec{V}_{0}+A \vec{h}_{0}\right) \times \vec{\delta}_{2}\right]=0
$$


или

$$
\begin{aligned}
& \frac{\partial \gamma_{0}^{(1)}}{\partial \tau}=\delta_{1} \gamma_{1}^{1}+\delta_{2} \gamma_{1}^{0} \\
& \frac{\partial \gamma_{1}^{(1)}}{\partial \tau}=-\delta_{1} \gamma_{0}^{1}-\delta_{2}\left(A+\gamma_{0}^{0}\right)
\end{aligned}
$$

Рассмотрим вспомогательную однородную систему

$$
\begin{aligned}
& \frac{\partial \widetilde{\gamma}_{0}^{(1)}}{\partial \tau}=\delta_{1} \widetilde{\gamma}_{1}^{1}, \\
& \frac{\partial \widetilde{\gamma}_{1}^{(1)}}{\partial \tau}=-\delta_{1} \widetilde{\gamma}_{0}^{1} .
\end{aligned}
$$

Очевидно, что

$$
\left(\widetilde{\gamma}_{0}^{(1)}\right)^{2}+\left(\widetilde{\gamma}_{1}^{(1)}\right)^{2}=\widetilde{C}_{0}^{2}(\xi, \eta)
$$

откуда

$$
\begin{aligned}
& \widetilde{\gamma}_{0}^{(1)}=\widetilde{C}_{0} \cos \tilde{\varphi}(\xi, \eta, \tau), \\
& \widetilde{\gamma}_{1}^{(1)}=\widetilde{C}_{0} \sin \tilde{\varphi}(\xi, \eta, \tau) .
\end{aligned}
$$

Подстановка этих формул в (15a) дает $\delta_{1}=-\partial \tilde{\varphi} / \partial \tau$. Учитывая выражение (12), получаем

$$
\tilde{\varphi}=\varphi(\xi, \eta, \tau)+\varphi_{0}(\xi, \eta)
$$

Ввиду этого будем искать решения системы (15) в виде

$$
\begin{aligned}
\gamma_{0}^{(1)}(\xi, \eta, \tau) & =\widetilde{\widetilde{C}}(\xi, \eta, \tau) \cos \tilde{\varphi}(\xi, \eta, \tau), \\
\gamma_{1}^{(1)}(\xi, \eta, \tau) & =\widetilde{\widetilde{C}}(\xi, \eta, \tau) \sin \tilde{\varphi}(\xi, \eta, \tau) .
\end{aligned}
$$

Получим

$$
\begin{gathered}
\cos \tilde{\varphi} \frac{\partial \widetilde{\widetilde{C}}}{\partial \tau}=\delta_{2} \gamma_{1}^{0}, \\
\sin \tilde{\varphi} \frac{\partial \widetilde{\widetilde{C}}}{\partial \tau}=-\delta_{2}\left(A+\gamma_{0}^{0}\right) .
\end{gathered}
$$

Умножая первое уравнение на $A+\gamma_{0}^{0}=C_{0} \cos \varphi$, второе - на правую часть первого и складывая результаты, получим

$$
C_{0} \cos (\tilde{\varphi}-\varphi) \frac{\partial \widetilde{\widetilde{C}}}{\partial \tau}=0
$$

или

$$
C_{0} \cos \varphi_{0} \frac{\partial \widetilde{\widetilde{C}}}{\partial \tau}=0
$$


Следовательно,

$$
\begin{gathered}
\delta_{2}=0, \\
\widetilde{C}^{2}(\xi, \eta)=\left(\gamma_{1}^{(1)}(\xi, \eta, 0)\right)^{2}+\left(\gamma_{0}^{(1)}(\xi, \eta, 0)\right)^{2} .
\end{gathered}
$$

Но начальные условия для членов высшего порядка асимптотического разложения (5) являются нулевыми. Следовательно, $\widetilde{C}_{0}(\xi, \eta) \equiv 0$, и все члены асимптотического разложения в виде (6) для $n>1$ обращаются в нуль.

Однако $\vec{V}_{0}+\varepsilon\left(\vec{\nu}_{1}+\vec{\delta}_{1}\right)$ не является точным решением системы (4). Следовательно, предположение о том, что $\varepsilon=R^{-1 / \kappa}$ для $\kappa>2$, означает отсутствие решений системы (4) в виде (6).

Положим $\kappa=2$, т. е. $\varepsilon=R^{-1 / 2}$. Тогда в $\varepsilon^{2}$-приближении в выражении (6) появляется $(1 / R) \Delta_{z}\left(\operatorname{rot}_{z} \vec{V}\right)$, и мы получаем следующее уравнение для $\vec{w}_{1}$ :

$$
\frac{\partial w}{\partial \tau}+\left[\left(\vec{V}_{0}+A b_{0} \vec{h}_{0}\right) \times \vec{\delta}_{2}\right]+\left[\vec{w}_{1} \times \vec{\delta}_{1}\right]+\vec{V}_{0}=0
$$

или

$$
\begin{aligned}
& \frac{\partial \gamma_{0}^{(1)}}{\partial \tau}=\delta_{1} \gamma_{1}^{1}+\delta_{2} \gamma_{1}^{0}-\gamma_{0}^{0}, \\
& \frac{\partial \gamma_{1}^{(1)}}{\partial \tau}=-\delta_{1} \gamma_{0}^{1}+\delta_{2}\left(A+\gamma_{0}^{0}\right)-\gamma_{1}^{0} .
\end{aligned}
$$

Рассмотрим вспомогательную систему

$$
\begin{aligned}
& \frac{\partial \widetilde{\gamma}_{0}^{(1)}}{\partial \tau}=\delta_{1} \widetilde{\gamma}_{1}^{1}, \\
& \frac{\partial \widetilde{\gamma}_{1}^{(1)}}{\partial \tau}=-\delta_{1} \widetilde{\gamma}_{0}^{1} .
\end{aligned}
$$

Очевидно, что

$$
\begin{gathered}
\left(\widetilde{\gamma}_{0}^{(1)}\right)^{2}+\left(\widetilde{\gamma}_{1}^{(1)}\right)^{2}=\widetilde{C}_{0}^{2}(\xi, \eta), \\
\widetilde{\gamma}_{0}^{(1)}=\widetilde{C}_{0} \cos \tilde{\varphi}(\xi, \eta, \tau), \\
\widetilde{\gamma}_{1}^{(1)}=\widetilde{C}_{0} \sin \tilde{\varphi}(\xi, \eta, \tau) .
\end{gathered}
$$

Подставляя в эти выражения (15б), получаем $\delta_{1}=-\partial \tilde{\varphi} / \partial \tau$. Учитывая формулу $(12)$, имеем $\tilde{\varphi}=\varphi(\xi, \eta, \tau)+\varphi_{0}(\xi, \eta)$. В силу этого мы будем искать решения (15б) в виде

$$
\begin{aligned}
\gamma_{0}^{(1)}(\xi, \eta, \tau) & =\widetilde{\widetilde{C}}(\xi, \eta, \tau) \cos \tilde{\varphi}(\xi, \eta, \tau), \\
\gamma_{1}^{(1)}(\xi, \eta, \tau) & =\widetilde{\widetilde{C}}(\xi, \eta, \tau) \sin \tilde{\varphi}(\xi, \eta, \tau) .
\end{aligned}
$$

Получаем

$$
\begin{gathered}
\cos \tilde{\varphi} \frac{\partial \widetilde{\widetilde{C}}}{\partial \tau}=\delta_{2} \gamma_{1}^{0}-\gamma_{0}^{0}, \\
\sin \tilde{\varphi} \frac{\partial \widetilde{\widetilde{C}}}{\partial \tau}=-\gamma_{1}^{0}-\delta_{2}\left(A+\gamma_{0}^{0}\right) .
\end{gathered}
$$


Умножая первое уравнение на $A+\gamma_{0}^{0}=C_{0} \cos \varphi$, а второе - на $\gamma_{1}^{0}=C_{0} \sin \varphi$, получаем

$$
C_{0} \cos \left(\tilde{\varphi}-\varphi_{0}\right) \frac{\partial \widetilde{\widetilde{C}}}{\partial \tau}=-\left[\left(\gamma_{1}^{0}\right)^{2}+\left(A+\gamma_{0}^{0}\right)^{2}\right]+A\left(A+\gamma_{0}^{0}\right)
$$

или

$$
C_{0} \cos \varphi_{0} \frac{\partial \widetilde{\widetilde{C}}}{\partial \tau}=-C_{0}^{2}+A C_{0} \cos \varphi
$$

Однако $\cos \varphi_{0}>0$ (малое начальное возмущение), и

$$
\frac{\partial \widetilde{\widetilde{C}}}{\partial \tau}=-\frac{C_{0}}{\cos \varphi_{0}}+\frac{A \cos \varphi}{\cos \varphi_{0}}<0,
$$

т. е. $\widetilde{\widetilde{C}}(\xi, \eta, \tau)$ является ограниченной убывающей функцией. Стало быть, величиной $\vec{w}_{1}$ можно пренебречь в отличие от $\delta_{1}$, экспоненциально растущей во времени.

Таким образом, асимптотическая процедура для $\varepsilon^{0}-$ и $\varepsilon^{1}$-приближений завершена. Очевидно, она может быть последовательно доведена до любого $n$, при этом уравнения (9) и (13) для $n>1$ будут включать $\gamma_{k}^{(0)}, \gamma_{k}^{(1)}, \delta_{k+1}$ для $k<n$ как известные свободные члены, поскольку они были найдены на предыдущих этапах асимптотической процедуры.

Теперь, учитывая выражение (8), получаем

$$
\begin{aligned}
\vec{V} \sim \vec{V}_{0}+\varepsilon\left(\vec{\delta}_{1}+\vec{\vartheta}_{1}\right)=\gamma_{0}^{0}(\xi, \eta, \tau) \vec{h}_{0}(z)+\gamma_{1}^{(1)}(\xi, \eta, \tau) \vec{h}_{1}(z)+ \\
\quad+\varepsilon\left(\begin{array}{c}
0 \\
0 \\
0 \\
\delta_{1}(\xi, \eta, \tau)
\end{array}\right)+\varepsilon\left(\begin{array}{c}
0 \\
\left(-\frac{\partial \gamma_{1}^{(0)}}{\partial \eta}+\frac{\partial \gamma_{0}^{(0)}}{\partial \xi}\right) \cos z-\left(\frac{\partial \gamma_{0}^{(0)}}{\partial \eta}+\frac{\partial \gamma_{1}^{(0)}}{\partial \xi}\right) \sin z
\end{array}\right)
\end{aligned}
$$

где $\xi=x \varepsilon, \eta=y \varepsilon, \tau=t \varepsilon, \varepsilon=R^{-1 / 2}$ и

$$
\vec{h}_{0}(z)=\left(\begin{array}{c}
\sin z \\
\cos z \\
0
\end{array}\right), \quad \vec{h}_{1}(z)=\left(\begin{array}{c}
\cos z \\
-\sin z \\
0
\end{array}\right) \text {. }
$$

Следовательно,

$$
\begin{aligned}
\vec{u}= & \vec{u}_{0}+\widetilde{V} \sim C_{0}\left(\begin{array}{c}
\sin (\varphi(\xi, \eta, \tau)+z) \\
\cos (\varphi(\xi, \eta, \tau)+z) \\
0
\end{array}\right)-R^{-1 / 2} C_{0}\left(\begin{array}{c}
0 \\
0 \\
\frac{\partial \varphi}{\partial \eta} \cos (\varphi+z)+\frac{\partial \varphi}{\partial \xi} \sin (\varphi+z)
\end{array}\right)+ \\
& +R^{-1 / 2}\left(\begin{array}{c}
0 \\
0 \\
\frac{\partial C_{0}}{\partial \xi} \cos (z+\varphi)-\frac{\partial C_{0}}{\partial \eta} \sin (z+\varphi)-\frac{\partial \varphi}{\partial \tau}
\end{array}\right),
\end{aligned}
$$

где $\xi=x R^{-1 / 2}, \eta=y R^{-1 / 2}, \tau=t R^{-1 / 2}$.

Первый член в выражении (17) может быть назван когерентной парой Бельтрами, поскольку он является линейной комбинацией двух промодулированных в плоскости $(x, y)$ течений Бельтрами $\vec{h}_{0}(z)$ и $\vec{h}_{1}(z)$, являющихся собственными векторами 
оператора ротора с собственным значением 1 и обменивающихся энергией в соответствии с эволюцией в каждой точке плоскости $(x, y)$ глобальной фазовой функции $\varphi(x / \sqrt{R}, y / \sqrt{R}, t / \sqrt{R})$. Так, при $\varphi=\pi / 2+2 \pi \kappa$ первый член выражения (17) превращается в $C_{0} \vec{h}_{0}(z)$, а при $\varphi=(2 \kappa+1) \pi$ он становится равным $C_{0} \vec{h}_{1}(z)$.

Первый член в выражении (17) постоянен по абсолютной величине для $t \sim R^{1 / 2}$, но он начинает уменьшаться на временах $t>R$ из-за наличия множителя $e^{-t / R}$ в уравнении (2), в то время как последний член в выражении (17) будет экспоненциально расти при $\tau \sim t / \sqrt{R}$ в силу того, что $\varphi$ является решением уравнения (14). Для выяснения поведения этого члена на очень больших временах $t \gg R$ введем новую шкалу времени, оставляя пространственнный скейлинг (3) неизменным:

$$
\frac{\partial}{\partial t} \rightarrow \varepsilon_{1} \frac{\partial}{\partial \tau_{1}}, \quad \tau_{1}=\varepsilon_{1} t, \quad \varepsilon_{1} \ll \frac{1}{R} .
$$

Рассматривая разложение (5) по степеням $\varepsilon$, мы следуем описанной выше процедуре.

Поскольку все члены, содержащие $e^{\varepsilon \tau}$, исчезают на временах $\tau \sim \tau_{1}, e^{-\varepsilon \tau_{1}}=$ $e^{-\varepsilon \varepsilon_{1} t} \rightarrow 0$, то, учитывая $\varepsilon=R^{-1 / 2}$, получаем

$$
\begin{gathered}
\operatorname{rot}_{z}\left[\operatorname{rot}_{z} \vec{V} \times \vec{V}\right]+\varepsilon\left\{\operatorname{rot}_{\xi \eta}\left[\operatorname{rot}_{z} \vec{V} \times \vec{V}\right]+\operatorname{rot}_{z}\left[\operatorname{rot}_{\xi \eta} \vec{V} \times \vec{V}\right]\right\}+ \\
+\varepsilon^{2}\left\{\operatorname{rot}_{\xi \eta}\left[\operatorname{rot}_{\xi \eta} \vec{V} \times \vec{V}\right] \Delta_{z} \operatorname{rot}_{z} \vec{V}\right\}-\varepsilon^{3} \operatorname{rot}_{\xi \eta} \Delta_{z} \vec{V}+\varepsilon^{4} \operatorname{rot}_{z} \Delta_{\xi \eta} \vec{V}- \\
-\varepsilon^{5} \operatorname{rot}_{\xi \eta} \Delta_{\xi \eta} \vec{V}+\varepsilon_{1} \frac{\partial}{\partial \tau_{1}}\left(\operatorname{rot}_{z} \vec{V}\right)+\varepsilon \varepsilon_{1} \frac{\partial}{\partial \tau_{1}} \operatorname{rot}_{\xi \eta} \vec{V}=0, \\
\operatorname{div}_{z} \vec{V}+\varepsilon \operatorname{div}_{\xi \eta} \vec{V}=0, \\
\varepsilon_{1} \frac{\partial}{\partial \tau_{1}} \int_{0}^{2 \pi} \vec{V} d z+\varepsilon \int_{0}^{2 \pi}\left[\operatorname{rot}_{\xi \eta} \vec{V} \times \vec{V}\right] d z+\varepsilon^{4} \int \Delta_{\xi \eta} \vec{V} d z=0 .
\end{gathered}
$$

С помощью асимптотической процедуры легко получить

$$
\vec{V}=\left(\begin{array}{c}
0 \\
0 \\
V_{0}^{(3)}\left(\varepsilon, \eta, \tau_{1}\right)
\end{array}\right),
$$

поэтому (опуская $\operatorname{rot}_{\xi \eta}$ )

или

$$
\varepsilon \varepsilon_{1} \frac{\partial}{\partial \tau_{10}} \vec{V}=\varepsilon^{5} \Delta_{\xi \eta} \vec{V}_{0},
$$

$$
\varepsilon_{1}=\varepsilon^{4}=R^{-2} \text {. }
$$

Следовательно,

$$
\frac{\partial V_{0}^{(3)}}{\partial \tau_{1}}=\Delta_{\xi \eta} V_{0}^{(3)} .
$$

Начальным условием для этого выражения с периодическими граничными условиями является последний член выражения (17):

$$
V_{0}^{(3)}(\xi, \eta, 0)=\left.\frac{\partial \varphi}{\partial \tau}\right|_{\tau \sim 1} .
$$


Это параболическое уравнение, ограниченные решения которого экспоненциально убывают во времени: $\tau_{1}=1 / R^{2}$. Таким образом, длинноволновые возмущения, являющиеся решениями уравнения (2), затухают при $t \sim R^{2}$.

\section{3. АСИМПТОТИЧЕСКОЕ ПОВЕДЕНИЕ ЛИНИЙ ТОКА}

Поскольку в силу первых членов выражения (17) выясняется,что

$$
\frac{d \varphi(\xi(t), \eta(\tau), \tau)}{d t}=\frac{\partial \varphi}{\partial \xi} \dot{\xi}(\tau)+\frac{\partial \varphi}{\partial \eta} \dot{\eta}(\tau)+\frac{\partial \varphi}{\partial \tau}=C_{0}\left(\frac{\partial \varphi}{\partial \xi} \sin (\varphi+z)+\frac{\partial \varphi}{\partial \eta} \cos (\varphi+z)\right)+\frac{\partial \varphi}{\partial \tau}
$$

линии тока поля скоростей (17) являются траекториями следущей системы обыкновенных дифференциальных уравнений:

$$
\begin{gathered}
\dot{\xi}=C_{0} \sin (\varphi(\xi, \eta, \tau)+z), \\
\dot{\eta}=C_{0} \cos (\varphi(\xi, \eta, \tau)+z), \\
\frac{d(z+\varphi)}{d \tau}=\frac{\partial C_{0}}{\partial \xi} \cos (z+\varphi)-\frac{\partial C_{0}}{\partial \eta} \sin (z+\varphi), \\
\xi(0)=\xi_{0}, \quad \eta(0)=\eta_{0}, \quad z(0)=z_{0},
\end{gathered}
$$

где $\varphi(\xi, \eta, \tau)$ определяется из задачи Коши (14):

$$
\begin{gathered}
\frac{\partial^{2} \varphi}{\partial \tau^{2}}=-\frac{C_{0}^{2}(\xi, \eta)}{2}\left(\frac{\partial^{2} \varphi}{\partial \xi^{2}}+\frac{\partial^{2} \varphi}{\partial \eta^{2}}\right)+\frac{\partial C_{0}}{\partial \xi} \frac{\partial \varphi}{\partial \xi}+\frac{\partial C_{0}}{\partial \eta} \frac{\partial \varphi}{\partial \eta} \\
C_{0}^{2}(\xi, \eta)=\left(A b_{0}+\gamma_{0}^{0}(\xi, \eta)\right)^{2}+\gamma_{1}^{0}(\xi, \eta)^{2} \\
\varphi(\xi, \eta, 0)=\operatorname{arctg} \frac{\gamma_{1}^{0}(\xi, \eta)}{A b_{0}+\gamma_{0}^{0}(\xi, \eta)}=\varphi_{0}(\xi, \eta), \\
\left.\frac{\partial \varphi}{\partial \tau}\right|_{\tau=0}=\delta_{0}(\xi, \eta) .
\end{gathered}
$$

Здесь $\gamma_{0}(\xi, \eta)$ - начальное длинноволновое амплитудное возмущение $\overrightarrow{h_{0}(z)}$, а $\gamma_{1}(\xi, \eta)-$ начальное длинноволновое амплитудное возмущение $\overrightarrow{h_{1}(z)}$. Предположение о наличии "длинноволнового возмущения" означает, что разложение функций $\gamma_{0}^{(0)}(\xi, \eta)$, $\gamma_{1}^{(0)}(\xi, \eta), \delta_{0}(\xi, \eta)$ в двойной ряд Фурье содержит конечное число членов. В первоначальных переменных уравнения (18) для вторичного течения, порожденного длинноволновой неустойчивостью нестационарного течения Тркаля, принимают вид

$$
\begin{aligned}
& \dot{x}=C_{0}\left(\frac{x}{\sqrt{R}}, \frac{y}{\sqrt{R}}\right) \sin \left(z+\varphi\left(\frac{x}{\sqrt{R}}, \frac{y}{\sqrt{R}}, \frac{t}{\sqrt{R}}\right)\right), \\
& \dot{y}=C_{0}\left(\frac{x}{\sqrt{R}}, \frac{y}{\sqrt{R}}\right) \cos \left(z+\varphi\left(\frac{x}{\sqrt{R}}, \frac{y}{\sqrt{R}}, \frac{t}{\sqrt{R}}\right)\right), \\
& \frac{d(z+\varphi)}{d t}=\left(\frac{\partial C_{0}}{\partial x} \cos (z+\varphi)-\frac{\partial C_{0}}{\partial y} \sin (z+\varphi)\right) \frac{1}{\sqrt{R}},
\end{aligned}
$$

где $C_{0}$ и $\varphi$ определены в системе (14). 
Введем величину $w=z+\varphi$ Назовем квазистационарными решения $\bar{w}$ третьего уравнения системы (18), удовлетворяющие условию $d \bar{w} / d \tau=0$. Тогда

$$
0=\frac{d(\bar{z}+\varphi(\bar{\xi}, \bar{\eta}, \tau))}{d \tau}=\frac{\partial C_{0}(\bar{\xi}, \bar{\eta})}{\partial \xi} \cos (\bar{z}+\varphi(\bar{\xi}, \bar{\eta}, \tau))-\frac{\partial C_{0}(\bar{\xi}, \bar{\eta})}{\partial \eta} \sin (\bar{z}+\varphi(\bar{\xi}, \bar{\eta}, \tau))
$$

т. e.

$$
\frac{\partial C_{0}(\bar{\xi}, \bar{\eta}) / \partial \xi}{\partial C_{0}(\bar{\xi}, \bar{\eta}) / \partial \eta}=\operatorname{tg}(\bar{z}+\varphi(\bar{\xi}, \bar{\eta}, \tau))
$$

В силу первого и второго уравнений системы (18) получаем

$$
\frac{d \bar{\xi}}{d \bar{\eta}}=\frac{\left(C_{0}(\bar{\xi}, \bar{\eta})\right)_{\xi}}{\left(C_{0}(\bar{\xi}, \bar{\eta})\right)_{\eta}}
$$

Это уравнение определяет (с начальными условиями) некую кривую в плоскости $(\xi, \eta)$, наклон которой равен наклону вектора градиента функции $C_{0}(\xi, \eta)$. Эта кривая является проекцией квазистационарной линии тока $(\bar{\xi}(\tau), \bar{\eta}(\tau), \bar{z}(\tau))$ на плоскость $(\xi, \eta)$, где

$$
\begin{gathered}
\bar{z}(\tau)=-\varphi(\bar{\xi}(\tau), \bar{\eta}(\tau), \tau)+C_{0}, \\
C_{0}=z_{0}+\varphi_{0}\left(\bar{\xi}_{0}, \bar{\eta}_{0}\right) .
\end{gathered}
$$

Функция $\varphi$ является решением системы (14), т. е. экспоненциально растет во времени $\tau$.

Легко показать, что $\bar{w}$ является устойчивым решением третьего уравнения системы (18). В самом деле, положим $w=\bar{w}+\widetilde{w}$, где $\widetilde{w}(\xi, \eta, z, \tau)$ - малая функция, $\xi=\bar{\xi}+\tilde{\xi}, \eta=\bar{\eta}+\tilde{\eta}$. Тогда линеаризованная система (18) принимает вид

$$
\begin{aligned}
\frac{d \tilde{\xi}}{d \tau}= & \left(\frac{\partial C_{0}}{\partial \xi} \tilde{\xi}+\frac{\partial C_{0}}{\partial \eta} \tilde{\eta}\right) \sin \bar{w}+\widetilde{w} C_{0}(\bar{\xi}, \bar{\eta}) \cos \bar{w} \\
\frac{d \tilde{\eta}}{d \tau}= & \left(\frac{\partial C_{0}}{\partial \xi} \tilde{\xi}+\frac{\partial C_{0}}{\partial \eta} \tilde{\eta}\right) \cos \bar{w}+\widetilde{w} C_{0}(\bar{\xi}, \bar{\eta}) \sin \bar{w} \\
\frac{d \widetilde{w}}{d \tau}= & \frac{\partial}{\partial \xi}\left(\frac{\partial C_{0}}{\partial \xi} \tilde{\xi}+\frac{\partial C_{0}}{\partial \eta} \tilde{\eta}\right) \cos \bar{w}-\frac{\partial}{\partial \eta}\left(\frac{\partial C_{0}}{\partial \xi} \tilde{\xi}+\frac{\partial C_{0}}{\partial \eta} \tilde{\eta}\right) \sin \bar{w}- \\
& -\widetilde{w}\left(\frac{\partial C_{0}}{\partial \xi} \sin \bar{w}+\frac{\partial C_{0}}{\partial \eta} \cos \bar{w}\right)
\end{aligned}
$$

Но нас интересуют векторы возмущения $(\tilde{\xi}(\tau), \tilde{\eta}(\tau), \widetilde{w}(\tau))$, ортогональные вектору $\operatorname{grad} C_{0}(\bar{\xi}, \bar{\eta})$, т. е. такие, что

$$
\left(\frac{\partial C_{0}}{\partial \xi}\right)_{\substack{\xi=\bar{\xi} \\ \eta=\bar{\eta}}} \tilde{\xi}+\left(\frac{\partial C_{0}}{\partial \eta}\right)_{\substack{\xi=\bar{\xi} \\ \eta=\bar{\eta}}} \tilde{\eta}=0
$$


Следовательно,

$$
\begin{gathered}
\frac{d \tilde{\xi}}{d \tau}=\widetilde{w} C_{0}(\bar{\xi}, \bar{\eta}) \cos \bar{w} \\
\frac{d \tilde{\eta}}{d \tau}=-\widetilde{w} C_{0}(\bar{\xi}, \bar{\eta}) \sin \bar{w} \\
\frac{d \widetilde{w}}{d \tau}=-\left[\frac{\partial C_{0}}{\partial \xi} \sin \bar{w}+\frac{\partial C_{0}}{\partial \eta} \cos \bar{w}\right] \widetilde{w}
\end{gathered}
$$

где

$$
\operatorname{tg} \bar{w}=\frac{\partial C_{0} / \partial \xi}{\partial C_{0} / \partial \eta}
$$

Это означает, что

$$
\begin{aligned}
\sin \bar{w} & =\frac{\partial C_{0}}{\partial \xi} \frac{1}{\sqrt{\left(\partial C_{0} / \partial \xi\right)^{2}+\left(\partial C_{0} / \partial \eta\right)^{2}}} \\
\cos \bar{w} & =\frac{\partial C_{0}}{\partial \eta} \frac{1}{\sqrt{\left(\partial C_{0} / \partial \xi\right)^{2}+\left(\partial C_{0} / \partial \eta\right)^{2}}}
\end{aligned}
$$

следовательно,

$$
\begin{gathered}
\frac{\partial C_{0}}{\partial \xi} \sin \bar{w}+\frac{\partial C_{0}}{\partial \eta} \cos \bar{w}=\sqrt{\left(\frac{\partial C_{0}}{\partial \xi}\right)^{2}+\left(\frac{\partial C_{0}}{\partial \xi}\right)^{2}} \\
\frac{d \widetilde{w}}{d \tau}=-\widetilde{w} \sqrt{\left(\frac{\partial C_{0}}{\partial \xi}\right)^{2}+\left(\frac{\partial C_{0}}{\partial \eta}\right)^{2}} .
\end{gathered}
$$

Поскольку в ограниченной области (вне произвольно малых окрестностей конечного числа точек, в которых $\operatorname{grad} C=0$ ) выполняется условие

$$
m \leqslant \sqrt{\left(\frac{\partial C_{0}}{\partial \xi}\right)^{2}+\left(\frac{\partial C_{0}}{\partial \xi}\right)^{2}} \leqslant M
$$

то

$$
\left|\widetilde{w}_{(t)}\right|=c_{0} e^{-\int_{\tau_{0}}^{\tau} \sqrt{\left(\partial C_{0} / \partial \xi\right)^{2}+\left(\partial C_{0} / \partial \xi\right)^{2}}} d \tilde{\tau} \leqslant C_{0} e^{-m\left(\tau-\tau_{0}\right)} .
$$

Следовательно, проекция линии тока на плоскость $(\xi, \eta)$ является кривой, асимптотически приближающейся к некой “предельной”, определяемой уравнениями для квазистационарных линий тока:

$$
\begin{aligned}
& \dot{\bar{\xi}}=C_{0} \frac{\partial C_{0}}{\partial \xi} \frac{1}{\left|\operatorname{grad} C_{0}(\bar{\xi}, \bar{\eta})\right|}, \\
& \dot{\bar{\eta}}=C_{0} \frac{\partial C_{0}}{\partial \eta} \frac{1}{\left|\operatorname{grad} C_{0}(\bar{\xi}, \bar{\eta})\right|},
\end{aligned}
$$

или, в векторной форме,

$$
\dot{\bar{\Sigma}}=C_{0}(\bar{\Sigma}) \frac{\operatorname{grad} C_{0}(\bar{\Sigma})}{\left|\operatorname{grad} C_{0}(\bar{\Sigma})\right|}
$$


где

$$
\bar{\Sigma}=\left(\begin{array}{c}
\bar{\xi} \\
\bar{\eta}
\end{array}\right)
$$

Таким образом, мы можем ввести стационарные "крупномасштабные структуры" как интегральные кривые поля градиента функции

$$
C_{0}(\xi, \eta)=\left[\left(A b_{0}+\gamma_{0}(\xi, \eta)\right)^{2}+\gamma_{1}^{2}(\xi, \eta)\right]^{1 / 2},
$$

где $\gamma_{0}(\xi, \eta)$ и $\gamma_{1}(\xi, \eta)$ - начальные малые длинноволновые амплитудные модуляции ("шум") двух течений Бельтрами

$$
\overrightarrow{h_{0}(z)}=\left(\begin{array}{c}
\sin z \\
\cos z \\
0
\end{array}\right), \quad \overrightarrow{h_{1}(z)}=\left(\begin{array}{c}
\cos z \\
-\sin z \\
0
\end{array}\right)
$$

являющихся собственными векторами оператора ротора с собственным значением 1.

Рассмотрим эволюцию функции $C_{0}(\bar{\xi}(\tau), \bar{\eta}(\tau))=C_{0}(\tau)$ вдоль квазистационарной линии тока $(\bar{\xi}(\tau), \bar{\eta}(\tau))$ :

$$
\begin{aligned}
\frac{d C_{0}(\tau)}{d \tau} & =\frac{\partial C}{\partial \xi} \dot{\bar{\xi}}(\tau)+\frac{\partial C}{\partial \eta} \dot{\bar{\eta}}(\tau)=\frac{\partial C}{\partial \xi} C_{0}(\bar{\xi}, \bar{\eta}) \sin \bar{w}+\frac{\partial C_{0}}{\partial \eta} C_{0}(\bar{\xi}, \bar{\eta}) \cos \bar{w}= \\
& =C_{0}(\bar{\xi}, \bar{\eta}) \sqrt{\left(\frac{\partial C_{0}}{\partial \xi}\right)^{2}+\left(\frac{\partial C_{0}}{\partial \eta}\right)^{2}},
\end{aligned}
$$

или

$$
\frac{d C_{0}(\tau) / d \tau}{C_{0}(\tau)}=\sqrt{\left(\frac{\partial C_{0}}{\partial \xi}\right)^{2}+\left(\frac{\partial C_{0}}{\partial \eta}\right)^{2}}
$$

Отсюда

$$
\left|C_{0}(\tau)\right|=c_{0} \exp \int_{0}^{\tau} \sqrt{\left(\frac{\partial C_{0}(\xi(t), \eta(t))}{\partial \xi}\right)^{2}+\left(\frac{\partial C_{0}(\xi(t), \eta(t))}{\partial \eta}\right)^{2}} d t .
$$

Поскольку $C_{0}(\xi, \eta)$ является ограниченной функцией во всей плоскости, из формулы (23) следует, что квазистационарная траектория может оставаться таковой вне любой заданной окрестности конечного числа "стационарных" точек, где градиент этой функции обращается в нуль, только конечное время.

Следовательно, интегральные линии соединяют “стационарные точки”, где градиент $C_{0}(\xi, \eta)$ обращается в нуль:

$$
\left|\operatorname{grad} C_{0}(\bar{\xi}, \bar{\eta})\right|=0,
$$

и “крупномасштабные структуры" образуются из этих устойчивых кривых $(t \sim \sqrt{R})$ в плоскости $(x, y)$.

Стационарные точки $C_{0}(\xi, \eta)$ являются либо точками максимума ("источниками"), либо точками минимума ("стоками"), либо седловыми точками. Каждая траектория начинается в какой-то точке максимума и кончается в какой-то точке минимума. Каждая седловая точка имеет одну входящую траекторию и одну выходящую - так называемые сепаратрисы. Сепаратрисы делят плоскость на области, 
состоящие из линий тока (точнее, их проекций), начинающихся в одной фиксированной критической точке и кончающихся в другой (иными словами, сепаратрисы делят плоскость на области гомотопных траекторий). Эти области инвариантны при движении жидкости.

Учитывая формулы (6), (8) и (17), легко видеть, что

$$
u-\operatorname{rot} u=\left(\frac{1}{R^{1 / 2}}\right) \vec{\delta}_{1}+O\left(\frac{1}{R}\right),
$$

где последнее выражение означает все члены асимптотического разложения высших порядков, а первый член в правой части является вектором, параллельным оси $z$.

Таким образом, в первом приближении (с точностью до членов порядка $1 / R$ ) вектор скорости $u$ и вектор ротора скорости rot $u$ лежат в касательной плоскости к вертикальной поверхности, проходящей через линию тока, определяемую уравнениями (21a). Заметим, что сказанное верно и для таких времен, когда первый член в правой части перестает быть малым, т.е. когда векторы скорости и ротора скорости уже, вообще говоря, могут не быть почти коллинеарными.

Следовательно, эту поверхность можно рассматривать и как “лист скоростей", и как "роторный лист". Листы скоростей, включающие все плоские трактории, соединяющие две фиксированные критические точки в плоскости, образуют “трубки скоростей", являющиеся инвариантными областями движения жидкости.

С точностью до членов порядка $1 / R$ "трубки скоростей" совпадают с "трубками ротора скорости", которые весьма устойчивы и при временах $\tau \sim 1, t \sim \sqrt{R}$.

\section{4. КАЧЕСТВЕННЫЕ (ТОПОЛОГИЧЕСКИЕ) ПРИМЕРЫ}

Для демонстрации концепции линий градиента приведем два простых примера.

Предположение о “длинноволновости” возмущений означает, что $\gamma_{0}(\xi, \eta)$ и $\gamma_{1}(\xi, \eta)$ являются двупериодическими функциями в плоскости $(\xi, \eta)$, иначе в любом представлении Фурье этих функций будут участвовать все пространственные частоты и понятие "длинноволновости" возмущения будет лишено смысла. Поэтому $\gamma_{0}(\xi, \eta)$ и $\gamma_{1}(\xi, \eta)$ являются конечными тригонометрическими полиномами двух переменных.

Мы ограничимся рассмотрением случая наличия в этих полиномах только первых гармоник. Поскольку $\gamma_{0}(\xi, \eta)$ и $\gamma_{1}(\xi, \eta)$ как "шумовые" начальные условия являются малыми, можно ввести параметр малости $\bar{\varepsilon}$, за который можно принять, например, наибольшее абсолютное значение обеих функций. Тогда

$C_{0}=\left[\left(A+\bar{\varepsilon} \bar{\gamma}_{0}(\xi, \eta)\right)^{2}+\bar{\varepsilon}^{2} \bar{\gamma}_{1}^{2}(\xi, \eta)\right]^{1 / 2}=\left[\left(A^{2}+2 \bar{\varepsilon} \bar{\gamma}_{0}(\xi, \eta)\right)\right]^{1 / 2}\left(1+\frac{\varepsilon^{2}}{2 A}\left(\bar{\gamma}_{0}^{2}+\bar{\gamma}_{1}^{2}\right)+\cdots\right)$.

Средствами алгебраической топологии можно показать [25], что для достаточно малых значений $\bar{\varepsilon}$ картина линий градиента для функции $C_{0}$ топологически эквивалентна картине линий градиента для функции $\widetilde{C}_{0}^{2}=A^{2}+2 \bar{\varepsilon} \bar{\gamma}_{0}(\xi, \eta)$. Поэтому важным становится вопрос о граничных условиях, так как сами стационарные точки, где градиент $\widetilde{C}_{0}$ (а стало быть, и градиент $\bar{\gamma}_{0}$ ) обращается в нуль, зависят от граничных условий для $\gamma_{0}(\xi, \eta)$. 


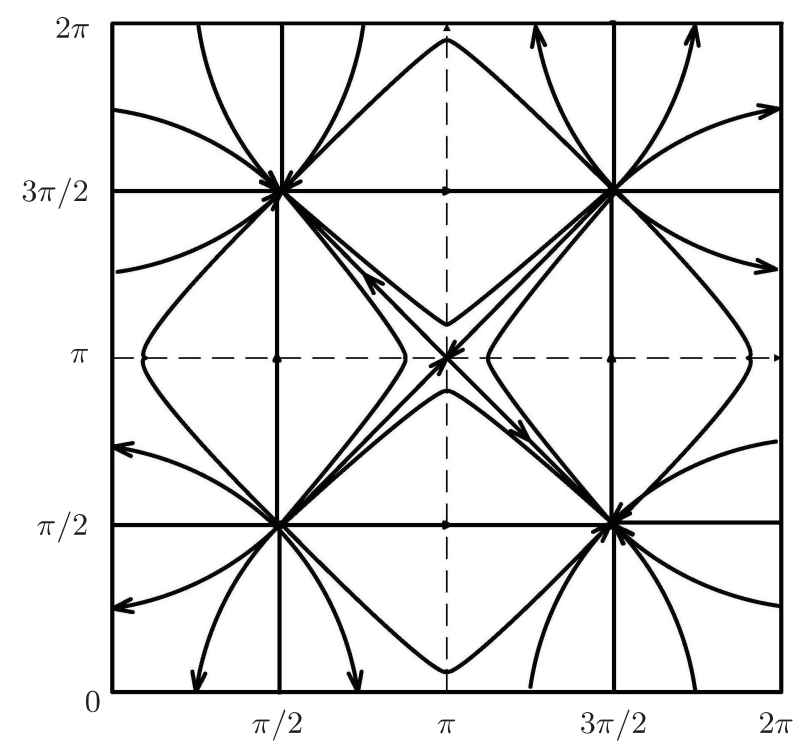

Рис. 1

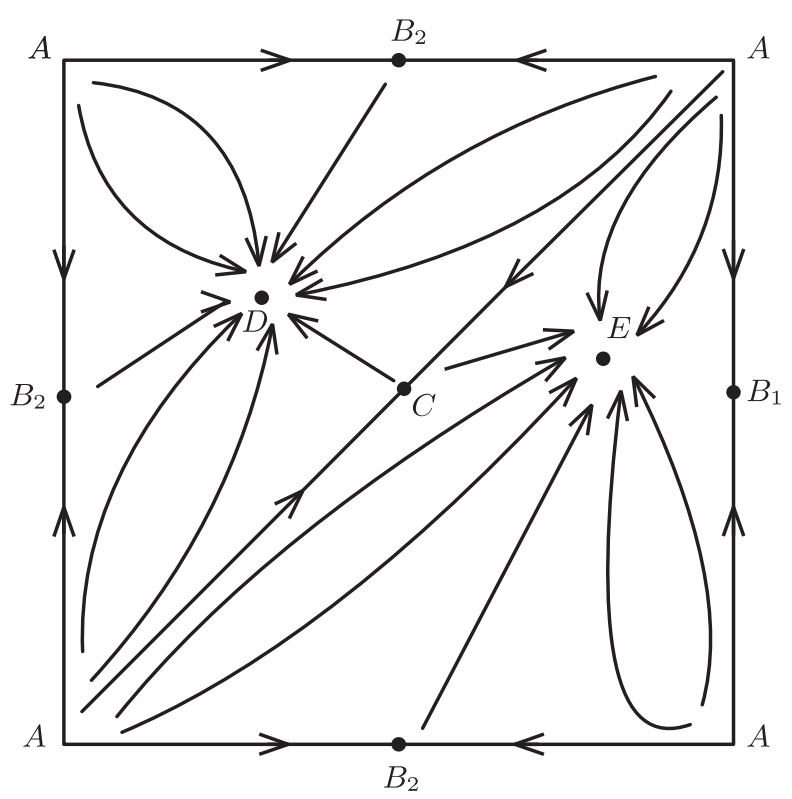

Рис. 2 


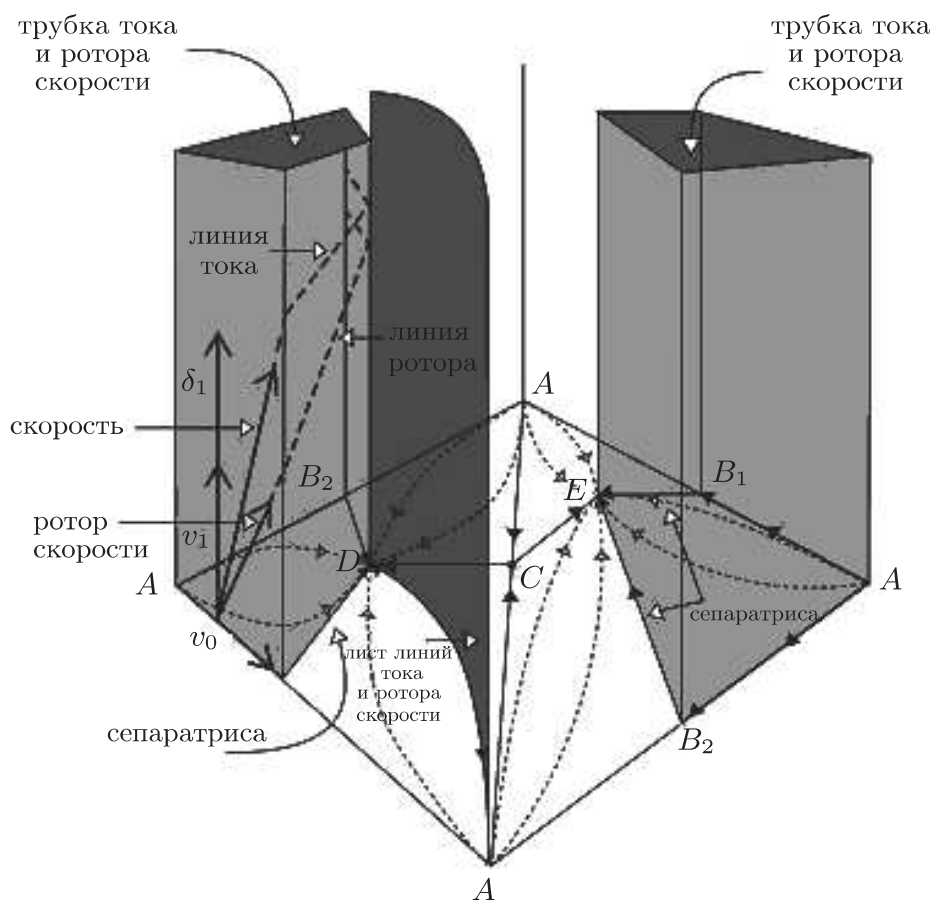

Рис. 3

Рассмотрим для иллюстрации случай, когда функция $\bar{\gamma}_{0}(\xi, \eta) 2 \pi$-периодична по $\xi$ и $\eta$ и обращается в нуль на границе квадрата $[0,2 \pi] \times[0,2 \pi]$. Очевидно, что $\bar{\gamma}_{0}(\xi, \eta)=$ $K \sin \xi \sin \eta$. Эта функция имеет 5 стационарных точек внутри квадрата (рис. 1): два максимума $(\pi / 2 ; \pi / 2)$ и $(3 \pi / 2 ; 3 \pi / 2)$, два минимума $(\pi / 2 ; 3 \pi / 2)$ и $(3 \pi / 2 ; \pi / 2)$ и одну седловую точку $(\pi, \pi)$. Точки максимума являются источниками, а точки минимума - стоками. Две сепаратрисы проходят через седловую точку.

Более содержательным является пример $2 \pi$-периодических граничных условий на границах квадрата $[0,2 \pi] \times[0,2 \pi]$ для функции $\gamma_{0}(\xi, \eta)$ (т. е. при рассмотрении $\gamma_{0}(\xi, \eta)$ на торе). Арнольд [26] выписывает такие функции в виде

$$
\bar{\gamma}_{0}(\xi, \eta)=a \cos \xi+b \sin \xi+c \cos \eta+d \sin \eta+p \cos (\xi+\eta)+q \sin (\xi+\eta)
$$

и доказывает, что, имея по шесть стационарных точек, эти функции допускают две различные топологические картины (по отношению к группе диффеоморфизмов тора) для линий уровня. Эти две картины, а с ними и картины линий градиента, определяются структурой шести стационарных точек: а) одной точки максимума, трех седловых точек и двух точек минимума; б) двух точек максимума, трех седловых точек и одной точки минимума.

Картина линий градиента для первого случая приведена на рис. 2.

Соответствующая трехмерная картина с некоторыми выделенными инвариантными областями ("роторно-скоростными трубками") показана на рис. 3. 


\section{5. ВЫВОДЫ}

Для исследования длинноволновых возмущений нестационарного анизотропного геликального решения нефорсированного уравнения Навье-Стокса мы использовали мультискейлинговый асимптотический анализ при больших числах Рейнольдса $R$, что позволило обнаружить специфические фигуры линий тока в плоскости, ортогональной анизотропному направлению. Эти фигуры оказались линиями градиента функции, определяемой начальными крупномасштабными амплитудными модуляциями двух течений Бельтрами с одинаковыми собственными значениями оператора ротора.

Спаривание этих модулированных течений Бельтрами ведет к возникновению квазистационарных фигур на временах порядка $R^{1 / 2}$, которые можно рассматривать как крупномасштабные структуры с характерным размером $R^{1 / 2}$. Общая картина определяется основными свойствами начальных условий и иногда может быть понятна без сложных вычислений. Области гомотопных плоских линий тока ограничены сепаратрисами, определяющими как инвариантные подмножества плоского течения, так и инвариантные трехмерные роторно-скоростные цилиндры (“трубки"), инвариантные для потоков поля скорости и поля ротора скорости и характиризуемые, как правило, асимптотической коллинеарностью векторов скорости и ротора скорости. Параллельная направлению анизотропии компонента трехмерного крупномасштабного вектора сторости неустойчива и может быть получена непосредственно как решение задачи Коши для уравнения эллиптичекого типа (типичный случай некорректно поставленной задачи), коэфиициенты которого определяются начальными условиями. Эта компонента скорости переживает течение Тркаля и затухает на временах порядка $R^{2}$.

Благодарности. Автор выражает глубокую благодарность профессору Е. Левичу за непрестанное содействие в ознакомлении с современными концепциями турбулентности и постоянное внимание к усилиям автора в написании настоящей статьи, а также профессору Л. Полтеровичу за многочисленные консультации и советы.

\section{Список литературы}

[1] E. Levich, A. Tsinober, Phys. Lett. A, 93:6 (1983), 293-297.

[2] E. Levich, E. Tzvetkov, Phys. Lett. A, 100:1 (1984), 53-56.

[3] E. Levich, Old and New Concepts Phys., 6:3 (2009), 239-457.

[4] A. Tsinober, E. Levich, Phys. Lett. A, 99:6-7 (1983), 321-324.

[5] H. K. Moffatt, J. Fluid Mech., 159 (1985), 359-378.

[6] H. K. Moffatt, J. Fluid Mech., 166 (1986), 359-378.

[7] H. K. Moffatt, "The topology of scalar fields in 2D and 3D turbulence", IUTAM Symposium on Geometry and Statistics of Turbulence, Fluid Mech. Appl., 59, eds. T. Kambe, T. Nakano, T. Miyauchi, Kluwer, Dordrecht, 2001, 13-22.

[8] V. Trkal, Časopis št. Mat., 48 (1919), 302-311.

[9] Я. Андреопулос, Электрохимия, 44:4 (2008), 422-428.

[10] Yeontaek Choi, Byong-Gu Kim, Changhoon Lee, Phys. Rev. E, 80:1 (2009), 017301, 4 pp.

[11] M. S. Lilley, S. Lovejoy, K. Strawbridge, D. Schertzer, A. Radkevich, Quart. J. Roy. Meteor. Soc., 134:631 (2008), 301-315. 
[12] S. Lovejoy, A. Tuck, S. Hovde D. Schertzer, Geophys. Res. Lett., 34:15 (2007), L15802.

[13] S. Lovejoy, D. Schertzer, M. Lilley, K. Strawbridge, A. Radkevich, Quart. J. Roy. Meteor. Soc., 134:631 (2008), 277-300.

[14] P. D. Mininni, A. Alexakis, A. Pouquet, Phys. Rev. E, 74:1 (2006), 016303, 13 pp., arXiv: physics/0602148v2.

[15] P. D. Mininni, A. Alexakis, A. Pouquet, Phys. Rev. E, 77:3 (2008), 036306, 9 pp., arXiv: $07809.1939 \mathrm{v} 1$.

[16] J. Molinari, D. Vollaro, Monthly Weather Rev., 136:11 (2008), 4355-4372.

[17] Annick Pouquet, Figures and videos, http://www.image.ucar.edu/ pouquet/Figs.htm.

[18] A. Radkevich, S. Lovejoy, K. Strawbridge, D. Schertzer, M. Lilley, Quart. J. Roy. Meteor. Soc., 134:631 (2008), 317-335.

[19] G. I. Sivashinsky, Physica D, 17:2 (1985), 243-255.

[20] A. Libin, G. I. Sivashinsky, Quart. Appl. Math., 48:4 (1990), 611-623.

[21] A. Libin, G. I. Sivashinsky, Phys. Lett. A, 144:3 (1990), 172-178.

[22] L. Shtilman, G. Sivashinsky, J. de Physique, 47:7 (1986), 1137-1140.

[23] V. Yakhot, G. Sivashinsky, Phys. Rev. A, 35:2 (1987), 815-820.

[24] A. Libin, G. I. Sivashinsky, E. Levich, Phys. Fluids, 30:10 (1987), 2984-2986.

[25] Л. Полтерович, Частное сообщение, 2009.

[26] В. И. Арнольд, "Топологическая классификация тригонометрических многочленов аффинной группы Коксетера $\widetilde{A}_{2} "$, Анализ и особенности. Часть 1, Сборник статей. K 70-летию со дня рождения академика Владимира Игоревича Арнольда, Тр. МИАН, 258, Наука, М., 2007, 7-16.

Поступила в редакцию 22.01.2010, после доработки 16.04.2010 